\title{
Takotsubo syndrome in association with pheochromocytoma: clinical and practical considerations
}

\author{
Kenan Yalta ${ }^{1}$, Ugur Ozkan ${ }^{1}$, Tulin Yalta ${ }^{2}$, Ertan Yetkin ${ }^{3}$ \\ ${ }^{1}$ Cardiology Department, Trakya University, Edirne; ${ }^{2}$ Trakya University, Pathology Department, Edirne; ${ }^{3}$ Derindere \\ Hospital, Cardiology Department, Istanbul, Turkey
}

Dear Editor,

In clinical practice, pathophysiology of Takotsubo syndrome (TTS) has been attributed to adrenergic discharge mostly associated with a variety of stressors [1-3]. Occasionally, organic sources of adrenergic discharge (including pheochromocytoma) might also account for this phenomenon, and are not considered as exclusion criteria for the diagnosis of TTS (as opposed to previous suggestions) $[1,2,4]$. We read with great interest the recently published article by Maffè et al. that describes a case of fatal TTS due to a ruptured pheochromocytoma in a middle-aged male [1]. In this regard, we would like to further analyse this interesting case and underscore potential implications of TTS associated with pheochromocytoma:

First, TTS episodes in the setting of pheochromocytoma usually emerge in the absence of overt stressors, and might have a significant predisposition to certain complications including malignant arrhythmogenesis, heart failure (HF) and coronary ischemic syndromes (associated with coronary microvascular dysfunction characterized by a coronary slow flow (CSF) pattern) [2-4].

Correspondence: Kenan Yalta, Cardiology Department, Trakya University, Edirne, Turkey.

Tel. +90 5056579856 .

E-mail: kyalta@gmail.com, akenanyalta@trakya.edu.tr.

Authors' contributions: KY: Design, conception, acquisition of literature data, analysis, interpretation, substantial writing, drafting, final revision and approval, affirming responsibility for the final content; UO: Design, conception, acquisition of literature data, analysis, interpretation, writing, drafting, final revision and approval, affirming responsibility for the final content; TY: Design, conception, acquisition of literature data, analysis, interpretation, writing, drafting, final revision and approval, affirming responsibility for the final content; EY: Design, conception, acquisition of literature data, analysis, interpretation, writing, drafting, final revision and approval, affirming responsibility for the final content.

Conflict of interest: The authors declare no potential conflict of interest.

Received for publication: 14 March 2021.

Accepted for publication: 27 April 2021.

${ }^{\circ}$ Copyright: the Author(s), 2021

Licensee PAGEPress, Italy

Monaldi Archives for Chest Disease 2021; 91:1848

doi: 10.4081 monaldi.2021.1848

This article is distributed under the terms of the Creative Commons Attribution Noncommercial License (by-nc 4.0) which permits any noncommercial use, distribution, and reproduction in any medium, provided the original author(s) and source are credited.
Accordingly, TTS episodes due to pheochromocytoma were reported to have significantly higher rates of in-hospital adverse events (mostly in relatively young patients) in a previous analysis on 80 published cases [4]. In particular, we hold the opinion that intractable ventricular arrhythmias along with a severe CSF pattern on invasive coronary angiogram (CAG) might be strongly indicative of a co-existing pheochromocytoma [2], and requires further investigation in patients with TTS (particularly in those without an overt TTS trigger). Therefore, did the authors detect any significant arrhythmias or dynamic electrocardiographic (ECG) findings (consistent with acute coronary ischemia)?

Second, pheochromocytoma was previously reported to be associated with chemical myocarditis (possibly due to excessive and prolonged adrenergic discharge) that might present with diffuse myocardial edema, and to some extent, irreversible myocardial damage (as best demonstrated with persistent late godolinium enhancement (LGE) on magnetic resonance imaging (MRI) and histopathological examination) [2]. Moreover, co-existence of TTS and chemical myocarditis might also be considered as a quite possible clinical scenario in the setting of pheochromocytoma [2]. In the present case, post-mortem examination of myocardium revealed histopathological findings of TTS including contraction band necrosis and neutrophilic infiltration [2]. Specifically, neutrophilic infiltration in the setting of TTS arises as an early histopathological finding, and is gradually replaced by the predominant infiltration of pro-inflammatory macrophages (M1 type) within the myocardium [2]. However, the patient had no macrophage infiltration of myocardium possibly due to his rapid deterioration resulting in early mortality [2]. Importantly, additional histopathological findings including disseminated myocardial damage and areas of myocardial infarction (attributable to severe microvascular dysfunction and/or direct catecholamine toxicity) might be considered as components of a co-existing chemical myocarditis in the patient [1].

Importantly, co-existence of TTS and chemical myocarditis might further worsen the in-hospital prognosis possibly due to extensive myocardial involvement (as might be exemplified by the present case) [1]. Moreover, TTS episodes due to pheochromocytoma might present with persistent myocardial wall motion abnormalities (and even progressive myocardial remodelling and advanced heart failure (HF) in the long-term) possibly as a consequence of co-existing chemical myocarditis [2]. In this context, this might indicate regular assessment of ventricular functions on imaging modalities after discharge and timely initiation of advanced HF therapy, where necessary. In particular, we wonder whether the patient had echocardiographic findings of diffuse myocardial edema (potentially manifesting as global and transient increases in myocardial wall thicknesses along with reduced compensatory hyperkinesis in the unaffected myocardial terrritories) 
that might suggest an existing myocarditis component [1]. Of note, sensitivity and negative predictive value of echocardiographic findings might be relatively low (as compared with those of MRI findings) to safely exclude a co-existing myocarditis in this particular context. However, these findings might possibly have a significant positive predictive value, and might confirm the diagnosis of chemical myocarditis, particularly in the presence of other diagnostic clues. Among such clues, very high levels of troponins ( $>10,000 \mathrm{ng} / \mathrm{L}$ at $3 \mathrm{~h}$ ) and severe left ventricular dysfunction (ejection fraction: $20 \%$ ) might be particularly indicative of a co-existing myocarditis in the patient [1].

Third, extreme adrenergic discharge associated with pheochromocytoma [4] might also induce variable degrees of systemic inflammation that might have important pathogenetic and prognostic implications in the setting of TTS [2]. In this regard, this might also suggest potential benefits of certain anti-inflammatory strategies [2]. On the other hand, it also seems likely that the acute abdomen (due to the rupture of pheochromocytoma and hepatic trauma) might have substantially contributed to the systemic inflammatory response (as quantified with a white blood cell (WBC) count of $35,000 / \mathrm{mm}^{3}$ ) in the patient [1]. In this regard, we wonder about the potential elevation of other inflammation parameters.

Fourth, atypical morphological variants were found to be relatively frequent among TTS cases due to pheochromocytoma based on a previous analysis (basal and global variants comprising $30 \%$ and $20 \%$ of the whole TTS population, respectively) [4]. Moreover, rapid transition of initial wall motion abnormalities to atypical myocardial regions (usually within days) was previously reported in the setting of TTS episodes associated with pheochromocytoma [4]. Therefore, extension of apical ballooning pattern to other myocardial territories, on top of chemical myocarditis, might have also contributed to the rapid clinical deterioration in the patient [1].

Lastly, it seems noteworthy that patients with pheochromocytoma might more likely incur recurrent TTS episodes due to intermittent bouts of spontaneous adrenergic discharge [4]. However, the patient had no remarkable clinical history potentially suggesting that his adrenal mass had been in a dormant state until the time of traumatic accident [1].

In summary, most cardiologists are generally inclined to consider pheochromocytoma exclusively as a potential cause of secondary hypertension. However, this phenomenon might also emerge as a potentially overlooked trigger of TTS $[1,4]$. In the setting of TTS, certain features including atypical demographic patterns (relatively young age, male gender), absence of overt stressors and signs of extreme adrenergic discharge (existing CSF on CAG, intractable arrhythmias) might particularly suggest a coexisting pheochromocytoma, and warrant further investigation [4]. More specifically, a significant myocarditis component might potentially accompany certain TTS episodes associated with pheochromocytoma [2]. Taken together, TTS in association with pheochromocytoma might have important diagnostic and prognostic implications. However, this needs to be established through further studies.

\section{References}

1. Maffè S, Dellavesa P, Paffoni $\mathrm{P}$, et al. Takotsubo syndrome and pheochromocytoma: an insidious combination. Monaldi Arch Chest Dis 2021;91:1711.

2. Yalta K, Yetkin E, Yalta T. Systemic inflammation in patients with Takotsubo syndrome: a review of mechanistic and clinical implications. Monaldi Arch Chest Dis 2021;91:1718.

3. Yalta K, Taylan G, Yalta T, et al. Takotsubo cardiomyopathy in the setting of multiple sclerosis: a multifaceted phenomenon with important implications. Monaldi Arch Chest Dis 2020;90:1420.

4. Y-Hassan S. Clinical features and outcome of pheochromocytoma-induced Takotsubo syndrome: Analysis of 80 published cases. Am J Cardiol 2016;117:1836-44. 